\title{
LOV conquers (sm)All GTPases
}

\section{Rachelle Kosoff ${ }^{1}$ and Jonathan Chernoff $^{2 *}$}

\author{
Addresses: ${ }^{1}$ Cancer Biology Program, University of Pennsylvania, 451 Curie Boulevard, Philadelphia, PA 19104, USA; ${ }^{2}$ Cancer Biology Program, \\ Fox Chase Cancer Center, W451, 333 Cottman Avenue, Philadelphia, PA 19111, USA \\ *Corresponding author: Jonathan Chernoff (j_chernoff@fccc.edu) \\ Fl000 Biology Reports 2010, 2:28 (doi:I0.34I0/B2-28)
}

The electronic version of this article is the complete one and can be found at: http://f $1000 . c o m /$ reports/biology/content $/ 2 / 28$

\begin{abstract}
Knowledge of spatial patterning of GTPases is critical to understanding protein function since subcellular localization is essential for normal protein function. In this paper, we discuss how Hahn's group has created a new type of genetically encoded, light-activated enzyme that allows precise temporal and spatial control of the small GTPase, Racl. This reagent enables this group to analyze the roles of effector proteins in Racl function and may be applicable to other signaling proteins.
\end{abstract}

\section{Introduction and context}

The ability to exert precise spatial and temporal control over enzymes in an in vitro cell culture setting has been a longtime obsession for many cell biologists, and with good reason. Such tools allow one to ask and answer many questions about protein function which are not easily addressed by other methods. The trick is how to make and use such tools. For many years, various 'photo-caged' constructs [1-4], which generally consist of a chemical moiety that blocks enzyme function until decomposed by light, have been employed. Such photo-caged enzymes are not without problems, however, as they typically comprise chemically modified enzymes that must then be microinjected into cells, with attendant issues regarding proper localization, dosing, stability, and half-life within cells. Also, activation requires irradiation with UV light, which is toxic to cells. A much better approach would be to genetically encode the photo-caged enzyme and perform photo-cleavage with shorter wavelengths, but how?

\section{Major recent advances}

Recently, unnatural amino acid derivatives protected with a photo-cleavable moiety have been used to label endogenous proteins, but this method is cumbersome, requiring one to rewire the cells' tRNA system such that the altered amino acid is properly handled in protein translation $[5,6]$. Also, it is not clear whether this strategy can be easily adapted to mammalian cells.
Hahn's group [7], which has previously constructed a number of elegant biosensors for small Rho-family GTPases, solves many of these problems by fusing a portion of phototropin-1 containing a light oxygen voltage (LOV) domain to the N-terminus of constitutively active Rac1. When fused in this manner, LOV interacts in the dark to sterically inhibit Rac1 from binding to its effector proteins [7]. Upon exposure to 458-nm light, the photo-activatable Rac1 (PA-Rac1) molecule unfolds and unleashes the active GTPase, which is now competent to bind effectors and propagate signals. Importantly, the process is reversible as removal of the light source results in resumption of the closed conformation and inhibition of PA-Rac1. Perhaps equally important, the strategy is potentially portable, at least to other small GTPases, as Hahn's group shows that a similar LOV-Cdc42 construct also can be manipulated, albeit with some fine-tuning, with non-toxic wavelengths of light.

In the first of back-to-back papers in Nature, Wu et al. [7] use a PA-Rac1 construct to explore a longstanding problem in the GTPase field: are particular effector subsets employed by Rac1 to carry out specific functions? They found that irradiation of PA-Rac1 produced abundant protrusions within seconds of exposure and that the degree of protrusive activity could be titrated according to the intensity of irradiation, directly linked 
to intensity of Rac1 activation. In addition, in some cell types, localized activation of Rac1 near the cell edge was accompanied by directional migration, with protrusions at the light-activated edge and retraction at the opposite pole. The Rac1-induced protrusions could be inhibited by a peptide inhibitor of the Rac-activated kinase, Pak1, but not by myosin inhibitors, whereas the opposite was true with regard to cell retraction. To determine the mechanism whereby localized activation of Rac1 could affect myosin activity and actin organization at the opposite pole, Wu et al. [7] co-expressed PA-Rac1 with a RhoA biosensor, thereby showing that activated Rac1 immediately inactivates RhoA in its vicinity. These results square with a large body of literature regarding the yinyang relationship between these two small GTPases [8].

Life, however, especially life at the cellular level, is never that simple. In an accompanying paper, Machacek et al. [9] further explore how the activities of these GTPases are coordinated during cellular protrusion. Exploiting their existing stable small GTPase biosensors, they report that activated RhoA is not confined to the trailing edge of the cell but instead is activated at the leading cell edge, proximal to and prior to activation of Cdc42 and Rac1. These results suggest that RhoA is involved in the earliest steps in cell protrusion and that $\mathrm{Cdc} 42$ and Rac1 are invoked later to reinforce or stabilize nascent protrusions. While these findings are not revolutionary, as activated RhoA has been previously sighted at the leading edge $[10,11]$, they go further than any before in terms of clarifying the spatial and temporal relationships between these critical GTPases.

While the PA-Rac1 system is, in our opinion, the current champion in this arena, other contenders have also risen to the challenge of GTPase photo-activation. For example, Harwood and Miller [12] developed a photoactivatable Cdc42 GTPase using a different technology to achieve spatiotemporal control of small GTPases. Their photo-caged GTPases have an alkylated small molecule with a HaloTag ligand positioned at a critical residue (Cys 18) in the GTP-binding pocket, rendering them inactive. The ligand attached to the small molecule enhances silencing by recruitment of the recombinant protein, HaloTag, resulting in steric hindrance and interference with normal protein interactions and GTP binding. Upon UV irradiation, this small photo-cage dissociates and Cdc42 GTP-binding capacity is restored. Since most Rho GTPases contain Cys at position 18 in the nucleotide-binding site, this strategy is likely to be generalizable to other family members. However, this approach, though elegant, retains the limitations of cell toxicity from UV irradiation, irreversible activation, and the need for microinjection.

\section{Future directions}

Where do we go from here? It would be wonderful if the approach of Hahn's group - perhaps we can call it 'the fusion of $\mathrm{LOV}^{\prime}$ - proves exportable to other proteins beyond the family of small GTPases as photo-activation in general is too important a tool to be limited to a few enzymes. It is not clear how easy this will be, but promising alternatives exist. The Rosen lab [13] recently employed another reversible light-activated genetically encoded system that takes advantage of the photosensory protein phytochrome B (PhyB) from Arabidopsis thaliana and its light-dependent ligand, a domain of phytochrome interacting factor-3 (Pif3). When each partner is fused to a given protein pair (in this case, Cdc42 bound to PhyB and its effector, Wiskott-Aldrich protein, bound to Pif3), red light induces rapid binding and activation, resulting in actin filament assembly required for cell growth and motility. This approach is likely to be adaptable to other proteins as Levskaya et al. [14] have made similar fusions to drive Tiam1, a Rac activator, to particular spots on the plasma membrane in a light-controlled fashion, resulting in local activation of this GTPase. Whether one system or the other proves the most adaptable, it is clear from these reports that we are at the dawn of a new era in precision control of enzyme activity in cells.

\section{Abbreviations}

LOV, light oxygen voltage; PA-Rac1, photo-activatable Rac1; PhyB, phytochrome B; Pif3, phytochrome interacting factor-3.

\section{Competing interests}

The authors declare that they have no competing interests.

\section{References}

I. Renner C, Moroder L: Azobenzene as conformational switch in model peptides. Chembiochem 2006, 7:868-78.

2. Schierling B, Noël AJ, Wende W, Hien LT, Volkov E, Kubareva E, Oretskaya T, Kokkinidis M, Römpp A, Spengler B, Pingoud A: Controlling the enzymatic activity of a restriction enzyme by light. Proc Natl Acad Sci U S A 2010, 107:136I-6.

3. Nguyen A, Rothman DM, Stehn J, Imperiali B, Yaffe MB: Caged phosphopeptides reveal a temporal role for I4-3-3 in GI arrest and S-phase checkpoint function. Nat Biotechnol 2004, 22:993-1000.

FI000 Factor 3.2 Recommended

Evaluated by Stephen Jackson 16 Aug 2004, Jane Endicott I3 Sep 2004 
4. Rothman DM, Shults MD, Imperiali B: Chemical approaches for investigating phosphorylation in signal transduction networks. Trends Cell Biol 2005, 15:502-10.

5. Lawrence DS: The preparation and in vivo applications of caged peptides and proteins. Curr Op Chem Biol 2005, 9:570-5.

6. Lemke EA, Summerer D, Geierstanger BH, Brittain SM, Schultz PG: Control of protein phosphorylation with a genetically encoded photocaged amino acid. Nat Chem Biol 2007, 3:76972.

FI000 Factor 9.0 Exceptional

Evaluated by Anthony Czarnik 30 Nov 2007

7. Wu YI, Frey D, Lungu OI, Jaehrig A, Schlichting I, Kuhlman B, Hahn KM: A genetically encoded photoactivatable Rac controls the motility of living cells. Nature 2009, 46 I: 104-8.

FI000 Factor 4.8 Must Read

Evaluated by Kenneth Yamada 16 Sep 2009, Rachel Buchsbaum 22 Sep 2009

8. Burridge K, Doughman R: Front and back by Rho and Rac. Nat Cell Biol 2006, 8:78I-2.

9. Machacek M, Hodgson L, Welch C, Elliott H, Pertz O, Nalbant $P$, Abell A, Johnson GL, Hahn KM, Danuser G: Coordination of Rho GTPase activities during cell protrusion. Nature 2009, 461:99-103.

FI000 Factor 6.4 Must Read

Evaluated by Silvio Gutkind 02 Sep 2009, Martin Humphries 16 Sep 2009
10. Pertz O, Hodgson L, Klemke RL, Hahn KM: Spatiotemporal dynamics of RhoA activity in migrating cells. Nature 2006, 440:1069-72.

F1000 Factor 3.3 Recommended

Evaluated by Alan Rick Horwitz 27 Mar 2006, Brian Kuhlman 20 Jun 2006, Andrius Kazlauskas II Dec 2006

II. Kurokawa K, Matsuda M: Localized RhoA activation as a requirement for the induction of membrane ruffling. Mol Biol Cell 2005, 16:4294-303.

FI000 Factor 3.0 Recommended Evaluated by Christopher Turner 17 Oct 2005

12. Harwood KR, Miller SC: Leveraging a small-molecule modification to enable the photoactivation of rho GTPases. Chembiochem 2009, I4:2855-7.

13. Leung DW, Otomo C, Chory J, Rosen MK: Genetically encoded photoswitching of actin assembly through the Cdc42-WASPArp2/3 complex pathway. Proc Natl Acad Sci U S A 2008, 105: 12797-802.

FI000 Factor 4.8 Must Read

Evaluated by Axel Nohturfft 08 Sep 2008, Mikael Akke 09 Jan 2009

14. Levskaya A, Weiner OD, Lim WA, Voigt CA: Spatiotemporal control of cell signalling using a light-switchable protein interaction. Nature 2009, 461:997-1001.

FI000 Factor 9.6 Exceptional

Evaluated by lan Macara 2I Sep 2009, Mikael Akke I3 Nov 2009 